\title{
CRUMPLED CUBES THAT ARE NOT HIGHLY COMPLEX
}

\author{
ROBERT J. DAVERMAN ${ }^{1}$
}

\begin{abstract}
It is proved that any crumpled $n$-cube $C, n>5$, whose wildness is contained in an ( $n-2)$-manifold $S$ in Bd $C$ must be of Type 2, which is to say that its wildness, though possibly complicated, is not incredibly complicated.
\end{abstract}

Wild embeddings of $(n-1)$-spheres in the $n$-sphere $S^{n}$ are often studied one side at a time, by identifying the two crumpled $n$-cubes bounded by such an embedded sphere. The complexities of a crumpled $n$-cube $C(n \geqslant 5)$, studied in [4] and elsewhere, have been categorized into certain types. Relatively crude distinctions among the types, sufficiently discriminating for this paper, can be made by checking how maps of the 2-cell $B^{2}$ into $C$ can be improved under approximation: for the all-inclusive Type 3, there exist arbitrarily close approximations $f: B^{2} \rightarrow C$ such that $f\left(B^{2}\right) \cap \mathrm{Bd} C$ is 1dimensional; for Type 2, there exist approximations $f$ such that $f\left(B^{2}\right) \cap \operatorname{Bd} C$ is 0-dimensional; and for Type 1, there exist approximations $f$ such that $f\left(B^{2}\right) \cap \mathrm{Bd} C$ is a tame 0 -dimensional subset of $\mathrm{Bd} C$. (See $\S 6$ of [4] for more formal but noninclusive definitions.)

There is a fundamental problem with this scheme, left unresolved by this note, for it has not been determined whether every crumpled $n$-cube is of Type 2, a question identified several years ago in [3]. At one time this problem seemed central to the theory of codimension one embeddings, in that, while it was unknown whether every embedding $f$ of $S^{n-1}$ in $S^{n}$ could be approximated by locally flat embeddings, it was known that $f$ could be so approximated provided that $f\left(S^{n-1}\right)$ bounded two crumpled $n$-cubes of Type $2(n \geqslant 5)$. Recently Ancel and Cannon proved that every embedding of an $(n-1)$-manifold in an $n$-manifold $(n \geqslant 5)$ can be approximated by locally flat embeddings [1], without any restriction upon the types of crumpled cubes involved. The problem of possible types persists, however, suggesting that there may be a gap in the present knowledge concerning the kinds of wildness that can arise. Among crumpled cube fanciers one can discern widespread sentiment that non-Type 2 examples do exist. In that spirit, the theorem

Received by the editors October 31, 1977 and, in revised form, December 15, 1977.

AMS (MOS) subject classifications (1970). Primary 57A35, 57A50; Secondary 57A15, 57A40.

Key words and phrases. Wild embedding, locally flat embedding, crumpled cube, closed $n$-cell-complement, 1-ULC.

'Research supported in part by the National Science Foundation MCS 76-07274 A01 R011052-09. 
stated in the abstract makes a distinction of sorts. The manifold nature of the wild set $S$ is crucial to the result proved here: another open question asks whether a crumpled $n$-cube $C(n \geqslant 5)$ is of Type 2 if $\mathrm{Bd} C$ is locally collared in $C$ modulo an $(n-2)$-dimensional set $X$. (The answer is affirmative for $(n-3)$-dimensional sets $X$-see the proof of [4, Proposition 9.8].)

In the course of the main argument, it is advantageous to work with a closed $n$-cell-complement, by which is meant a crumpled $n$-cube that can be embedded in $S^{n}$ as the closure of the complement of an $n$-cell. For $n \geqslant 5$ no distinction should be made since every crumpled $n$-cube can be so embedded [5].

Other terms and notation used here correspond to those of [4].

The lemma that follows, designed to provide an important approximation early in the proof of theorem, apparently never has found its way into a manuscript concerning the subject. The combination of general position and cutting methods endemic to this material can be employed to give an alternate proof, essentially simpler but much longer, that does not appeal to the relatively intricate results from [5].

Lemma. Suppose $C$ is a closed $n$-cell-complement in $S^{n}(n \geqslant 5)$ such that Bd $C$ is locally flat modulo an $(n-2)$-manifold $S \subset \mathrm{Bd} C$ and $S$ is locally flat in Bd $C$. Then $C$ is of Type 1.

Proof According to Theorem 3.3 of [5], $C$ is of Type 1 if and only if $\mathrm{Bd} C$ admits (curvilinear) PL triangulations $T$ of arbitrarily small mesh such that $C-T^{(2)}$ is 1-ULC, where $T^{(2)}$ denotes the 2-skeleton of $T$. Establishing this latter condition is relatively easy, for the flatness of $S$ in $\mathrm{Bd} C$ allows us to obtain a triangulation $T$ of small mesh such that $S \cap T^{(2)} \subset T^{(1)}$. By [6] any 1-complex in $S$ can be adjusted via a small isotopy of $S$ so that it is tame in $S^{n}$, and in the situation at hand, where $S$ is locally flat in $\mathrm{Bd} C$, one can easily perform this adjustment of $S \cap T^{(2)} \subset T^{(1)}$ by a small isotopy of Bd $C$. As a result, for this modified triangulation $T, S \cap T^{(2)}$ is a 1-complex tamely embedded in $S^{n}$. An elementary result [4, Proposition 2.5] states that, for a compact subset $X$ of $\mathrm{Bd} C, C-X$ is 1-ULC if the complements of $X$ in both $S^{n}$ and Bd $C$ are 1-ULC. Clearly then $C-\left(T^{(2)} \cap S\right)$ is 1-ULC. It follows that $C-T^{(2)}$ is 1-ULC since Bd $C$ is locally flat at each point of $T^{(2)}-S$.

THEOREM. If $C$ is a closed $n$-cell complement in $S^{n}(n \geqslant 5)$ such that $\mathrm{Bd} C$ is locally flat modulo an $(n-2)$-sphere $\mathrm{S} \subset \mathrm{Bd} C$, then for each map $f: B^{2} \rightarrow C$ and $\varepsilon>0$ there exists a map $g: B^{2} \rightarrow C$ such that $\rho(g, f)<\varepsilon$ and $g\left(B^{2}\right) \cap$ $\mathrm{Bd} C$ is 0-dimensional.

Proof. The fundamental step is to approximate $f$ by $g^{\prime}$ such that the diameter of each component of $g^{\prime}\left(B^{2}\right) \cap S$ is less than $\varepsilon$. With standard methods such maps can be successively approximated so that, for the limit map $g, g\left(B^{2}\right) \cap S$ is 0 -dimensional, after which, because $\mathrm{Bd} C$ is locally flat 
at points of Bd $C-S, g\left(B^{2}\right)$ can be slid off Bd $C-S$ to transform $g\left(B^{2}\right) \cap$ Bd $C$ into a 0-dimensional set.

For leverage we construct a simpler closed $n$-cell-complement $C^{\prime}$ containing $C$. In the $n$-cell $A=S^{n}-\operatorname{Int} C$ we name an $(n-1)$-cell $D$ such that $D \cap \operatorname{Bd} A=S$ (cone over $S$ from a point of $\operatorname{Int} A$ ). Applying the Ancel-Cannon Locally Flat Approximation Theorem [1], we improve $D$ to another $(n-1)$-cell $D^{\prime}$ such that $D^{\prime} \subset A, D^{\prime} \cap \operatorname{Bd} A=S$, and $D^{\prime}$ is locally flat modulo its boundary, and then we thicken $D^{\prime}$ to an $n$-cell $A^{\prime} \subset A$ such that $A^{\prime} \cap \operatorname{Bd} C=S$, Bd $A^{\prime}$ is locally flat modulo $S$, and $S$ is flat in $\mathrm{Bd} A^{\prime}$. By the Lemma, the crumpled $n$-cube $C^{\prime}=S^{n}-\operatorname{Int} A^{\prime}$ is of Type 1 .

It is a well-known, easily proved property of an arbitrary crumpled cube $C$ (see [4, Proposition 2.1]) that any map of $B^{2}$ into $C$ can be approximated by one such that the inverse image of $\mathrm{Bd} C$ is 0 -dimensional. Accordingly, there exists a close approximation $f_{1}: B^{2} \rightarrow C$ to $f$ such that $f_{1}\left(\partial B^{2}\right) \subset$ Int $C$ and $f_{1}^{-1}(\mathrm{Bd} C)$ is 0 -dimensional. All approximations to $f_{1}$ that follow can agree with $f_{1}$ on $\partial B^{2}$.

Let $K_{1}$ and $K_{2}$ denote the crumpled ( $\left.n-1\right)$-cubes in Bd $C$ having $S$ as their boundaries, and let $Z_{1}$ and $Z_{2}$ denote the closures of the components of $C^{\prime}-C$, where $K_{i} \subset Z_{i}(i=1,2)$. Since $K_{i}$ is an absolute retract [2], there exist retractions $r_{i}$ of $Z_{i}$ to $K_{i}(i=1,2)$.

Now we use the relative simplicity of $C^{\prime}$ to approximate the map $f_{1}$ : $B^{2} \rightarrow C \subset C^{\prime}$ by a map $f_{2}: B^{2} \rightarrow C^{\prime}$ such that $f_{2}\left(B^{2}\right) \cap S$ is 0 -dimensional (we only need that $C^{\prime}$ is better than Type 3, nowhere using the fact that it is of Type 1). With sufficient care, the map $f_{3}: B^{2} \rightarrow C$ obtained from $f_{2}$ by setting $f_{3}(x)=r_{i} f_{2}(x)$ if $f_{2}(x) \in Z_{i}(i=1,2)$ is also an approximation to $f_{1}$.

We set $X=f_{2}^{-1}(C)$ and note that $f_{3}\left(B^{2}-X\right) \subset$ Bd $C$. Furthermore, $B^{2}-$ $X$ is naturally decomposed into the union of two open sets $U_{i}=f_{2}^{-1}\left(Z_{i}-K_{i}\right)$ such that $f_{3}\left(U_{i}\right) \subset K_{i}(i=1,2)$. Again invoking Proposition 2.1 of [4] for the maps $f_{3}: U_{i} \rightarrow K_{i}$, we approximate $f_{3}$ by $f_{4}: B^{2} \rightarrow C$ such that $f_{4}\left|X=f_{3}\right| X=$ $f_{2} \mid X, f_{4}\left(U_{i}\right) \subset K_{i}$ and $U_{i} \cap f_{4}^{-1}(S)$ is 0-dimensional $(i=1,2)$.

Recall that $f_{2}\left(B^{2}\right) \cap S=f_{4}(X) \cap S$ is 0-dimensional. Accordingly, it can be covered by an open subset $W$ of Bd $C$ whose components have diameter less than $\varepsilon$, and there then exists another open subset $W^{\prime}$ of $\mathrm{Bd} C$ such that

$$
f_{4}(X) \cap S \subset W^{\prime} \subset \mathrm{Cl} W^{\prime} \subset W .
$$

Finally, in each $U_{i}$ we cover $U_{i} \cap f_{4}^{-1}(S)$ by disjoint open sets $V_{i}$ and $V_{i}^{\prime}$ such that $f_{4}\left(V_{i}\right) \subset W, f_{4}\left(V_{i}^{\prime}\right) \subset \mathrm{Bd} C-\mathrm{Cl} W^{\prime}$, and the image under $f_{4}$ of each component of $V_{i}^{\prime}$ has diameter less than $\varepsilon$. With standard general position methods, applied to $f_{4} \mid \cup_{i}\left(V_{i} \cup V_{i}^{\prime}\right), f_{4}$ can be approximated by a map $g^{\prime}$, where

$$
g^{\prime}\left|B^{2}-\bigcup_{i}\left(V_{i} \cup V_{i}^{\prime}\right)=f_{4}\right| B^{2}-\bigcup_{i}\left(V_{i} \cup V_{i}^{\prime}\right),
$$

such that $g^{\prime} \mid \cup_{i}\left(V_{i} \cup V_{i}^{\prime}\right)$ is an embedding into $\mathrm{Bd} C$ (remark: this is possible only in case $n \geqslant 6$; in case $n=5$, the image of the (countable) 
singular set can be kept off $S$, which is sufficient to accomplish the aims of this process), $g^{\prime}\left(V_{i}\right) \subset W, g^{\prime}\left(V_{i}^{\prime}\right)$ misses $f_{4}(X) \cap S$, and the image under $g^{\prime}$ of each component of $V_{i}^{\prime}$ has diameter less than $\varepsilon(i=1,2)$. It follows that every component $Q$ of $g^{\prime}\left(B^{2}\right) \cap S$ has diameter less than $\varepsilon$, for $Q$ must be either a subset of $W$ or a component of $S \cap g^{\prime}\left(V_{1}^{\prime} \cup V_{2}^{\prime}\right)$.

Local versions of this argument give the following variation to the Theorem:

Corollary. Suppose $C$ is a crumpled $n$-cube $(n \geqslant 5)$ and $S$ is an $(n-2)-$ manifold, possibly with boundary, in $\mathrm{Bd} C$ such that $\mathrm{Bd} C$ is locally collared in $C$ modulo $S$. Then $C$ is of Type 2.

\section{REFERENCES}

1. F. D. Ancel and J. W. Cannon, The locally flat approximation of cell-like embedding relations (to appear).

2. R. H. Bing, Retractions onto spheres, Amer. Math. Monthly 71 (1964), 481-484.

3. R. J. Daverman, Pushing an $(n-1)$-sphere in $S^{n}$ almost into its complement, Duke Math. J. 39 (1972), 719-723.

4.

5. —_ Every crumpled $n$-cube is a closed n-cell-complement, Michigan Math. J. 24 (1977), 225-241.

6. C. L. Seebeck III, Tame arcs on wild cells, Proc. Amer. Math. Soc. 29 (1971), 197-201.

Department of Mathematics, University of Tennessee, Knoxville, Tennessee 37916 